Anales de Geografía de la Universidad Complutense ISSN: 0211-9803

http://dx.doi.org/10.5209/AGUC.62480

\title{
Los Servicios Avanzados de Internet: Nuevas oportunidades para el desarrollo de los territorios rurales
}

\author{
Francisco Xosé Armas Quintá ${ }^{1}$; Rubén Camilo Lois González²; Xosé Carlos Macía Arce ${ }^{3}$ \\ Recibido: 8 de marzo del 2017/ Enviado a evaluar: 29 de mayo del 2017/ Aceptado: 18 de octubre del 2018
}

Resumen. Pese a la diversidad que existe en las áreas rurales europeas, es evidente que, en las dos últimas décadas, han tenido lugar cambios significativos en las mismas tanto desde el punto de vista social como económico. Aunque aún hay posturas en la literatura científica sobre la generalización de los problemas más comunes que afectan al medio rural, hoy día debe tenerse muy en cuenta que este ya no es visto como una simple superficie de producción. Las áreas rurales son espacios pluriactivos y multifuncionales que aportan valores de un elevado interés para la sociedad. Las tecnologías de la información y de la comunicación contribuyen a la instalación de nuevas actividades económicas en estos espacios, si bien se necesita tanto capital humano como iniciativas políticas para que esta realidad se propague a todo el medio rural europeo, especialmente a las áreas más periféricas en riesgo de abandono.

Palabras claves: Desarrollo rural; Sociedad de la Información; Nuevas Tecnologías; Pluriactividad; Servicios Avanzados de Internet.

\section{[en] Advanced Internet Services: New opportunities for rural territorial development}

\begin{abstract}
It is evident that, despite the existing diversity in European rural areas, there has been significant changes seen in them over the last two decades, both from a social and economic point of view. Although scientific literature still holds views generalising the most common problems affecting the rural areas, today it certainly must no longer be considered as a simple production surface. Rural areas are pluri-active and multifunctional spaces that provide values of high interest to society. Information and Communication Technologies contribute to the installation of new economic activities in these spaces, albeit both human capital and political initiatives are required to spread this reality throughout the European countryside, especially when it comes to the more peripheral areas at risk of abandonment.
\end{abstract}

Key words: Rural development, Information Society, New Technologies, pluriactivity, Advanced Internet Services.

1 Departamento de Didácticas Aplicadas. Universidade de Santiago de Compostela.

E-mail: francisco.armas@usc.ec

2 Departamento de Geografía. Universidade de Santiago de Compostela.

E-mail: rubencamilo.lois@usc.ec

3 Departamento de Didácticas Aplicadas. Universidade de Santiago de Compostela.

E-mail: carlos.macia@usc.ec 


\section{[fr] Les Services Avancés d'Internet: De nouvelles opportunités pour le dévelopment des territoires ruraux}

Résumé. Malgré la diversité qu'existe dans les zones rurales européennes, il est évidente que, dans les deux derniers décennies, ils ont eu lieu changements significatifs tant du point de vue social comme économique. Bien qu'il existe encore des positions dans la littérature scientifique sur la généralisation des problèmes les plus courants qui affectent au milieu rural, aujourd'hui il convient de tenir compte qu'il n'est pas plus vu comme une simple surface de production. Les zones rurales sont des espaces pluriactifs et multifonctionnels qu'apportent des valeurs d'un élevé intérêt pour la société. Les technologies de l'information et de la communication contribuent à l'installation de nouvelles activités économiques dans ces espaces, si bien se précise autant capital humain comme des initiatives politiques pour que cette réalité se propage à tout le moyen rural européen, spécialement aux zones les plus périphériques en risque d'abandon.

Mots clés: Développement rural; Société de l'Information; Nouvelles Technologies; Pluriactivité; Services Avancés d'Internet.

Cómo citar. Armas Quinta, F.X., Lois González, R.C., Macía Arce, X.C. (2018): Los Servicios Avanzados de Internet: Nuevas oportunidades para el desarrollo de los territorios rurales. Anales de Geografia de la Universidad Complutense, 38(2), 271-287.

Sumario. 1. Introducción. 2. Concepto y estrategias de desarrollo rural. 3. La renovación conceptual de la brecha digital. 4. Los Servicios Avanzados de Internet: oportunidades de desarrollo para el medio rural. 5. Reflexiones finales. 6. Bibliografía.

\section{Introducción}

El proceso de difusión de las tecnologías de la información y de la comunicación y el auge de la Sociedad Postindustrial, no ha sido ajeno a las áreas rurales. En las mismas se han registrado cambios considerables, tanto a nivel espacial, funcional como socioeconómico (Sancho y Reinoso, 2012) y han sido objeto de estudio de numerosas disciplinas interesadas en su análisis y evolución (Prados, 2009). El medio rural actual muestra una complejidad de usos y funciones que se contrapone con la polarización claramente definida entre campo y ciudad de la primera mitad del siglo XX (García Ramón et al., 1995).

Las variadas funciones y significados que han sido atribuidos al medio rural, han hecho del mismo un concepto complejo y ambiguo, siendo muy difícil tanto explicar por qué un espacio es rural o urbano, como trazar una línea en un mapa que separe a ambos (Woods, 2011). Ya no se puede hablar de una frontera de lo urbano y de lo rural sino de un "gradiente de urbanización" en lo que se refiere a la intensidad de la edificación, en la actividad y estructura económica, o en la movilidad de personas y mercancías (Roca, 2003). La sociedad rural y el espacio rural no deben ser vistos como algo indivisible, ya que la ruralidad está caracterizada por una multitud de espacios sociales que solapan una misma área geográfica (Cloke, 2006).

Hasta finales de los años ochenta, el medio rural se encajó en un paradigma de carácter desarrollista y productivista tradicional revelado en forma de modernización de la agricultura, pero en la actualidad, el sistema productivo del medio rural se ha 
reestructurado hasta el punto de que lo rural ya no es sinónimo de agrario (Rubio, 2010) adoptando patrones de desarrollo regionales propios de las áreas urbanas (Ward y Brown, 2009).

El concepto de lo rural ha pasado, en las dos últimas décadas, de ser sinónimo de tosco y atrasado a tener connotaciones positivas y asociarlo a la calidad (Sancho y Reinoso, 2012). El medio rural está comenzando a ser visto, ya no solo como una simple superficie de producción, sino como un espacio que aporta una serie de valores de elevado interés para la sociedad global (Armesto, 2005). En esta percepción han influido las nuevas relaciones campo-ciudad a distintos niveles (social, cultural, económico, ambiental) que ponen en valor lo rural entre los ciudadanos urbanos, al considerarlo como bien público que forma parte del patrimonio conjunto de la sociedad (Rubio, 2010).

Las áreas rurales europeas han experimentado profundos cambios en las últimas décadas, disminuyendo el empleo del sector agrícola y acogiendo a otras actividades distintas a las ligadas del sector primario. Estos cambios hacen que las políticas sectoriales dirigidas al apoyo del sector agrario sean cada vez más inapropiadas para impulsar su desarrollo, siendo más aptas aquellas que apuesten por inversiones estratégicas y el impulso de nuevas actividades (Ward y Brown, 2009).

Las nuevas tecnologías han regenerado la interconexión entre lo rural y lo urbano facilitando la instalación de nuevas actividades industriales y de servicios (Moyano, 2000). Las tecnologías de la información y de la comunicación son, sin duda, una oportunidad para impulsar procesos de desarrollo en el medio rural, sobre todo en aquellas áreas más periféricas. La difusión de las tecnologías de la información y de la comunicación pueden suponer oportunidades de desarrollo en las áreas rurales, pero no con un uso básico de Internet.

Es necesario avanzar más allá de la consulta de información, la comunicación y todos aquellos usos simples de la Red. Para impulsar procesos de desarrollo se necesita que la población sepa aprovechar al máximo las fortalezas de estas tecnologías (Pick y Azari, 2008) y que se haga un uso intensivo de sus servicios avanzados (Piñeira et al., 2014). La brecha digital tradicional es un concepto debe ser reevaluado (Selwyn, 2006) debido al notable incremento del número de usuarios de Internet y en particular, la conectividad móvil.

\section{Concepto y estrategias de desarrollo rural}

Los intentos de definición del medio rural tienen una gran tradición en los estudios geográficos y, en un principio, se vinculó al éxodo rural masivo y a la concentración de población en las ciudades y, más tarde, se consideró lo rural y lo urbano como un modelo polarizado con continuidad entre ambos (Paniagua y Hoggart, 2002). La definición de lo rural puede llegar a resultar confusa ya que las investigaciones sobre aspectos rurales multidisciplinares, parecen dar carta blanca para elaborar muchos tipos de investigaciones con este título (Muilo, 2010). El hecho de que existan distintas percepciones sobre los elementos naturales, los económicos o los culturales 
que caracterizan la ruralidad, fue lo que llevó a la gran diversidad de las definiciones propuestas (Entrena-Durán, 2012).

Han sido muchos los autores que han intentado, con un éxito limitado, definir y delimitar el medio rural (Cloke, 1977; Hoggart, 1990; Clout 1993; Cloke y Goodwin, 1993; Halfacree, 1993; Lázaro, 1995; Ceña, 1995; García Sanz, 1996; Lois, 2014), entre otros, aportando puntos de vista que, en ocasiones, presentan grandes diferencias (Rodríguez, 2005). Algunos relativizan la vigencia de la distinción urbano-rural (Camarero, 1993), otros defienden la ruptura rural-agrario (García Sanz, 1996), y también hay quienes consideran inútil o imposible cualquier intento de delimitar conceptualmente el término rural (Sancho, 1997; Bericat, 1993). Rubio (2010) afirma que cuando un medio rural no coincide con el modelo de ruralidad, nuestra percepción inicia un desplazamiento hacia la noción de lo urbano. Pero la diversidad creciente de las áreas rurales y la dificultad de establecer una delimitación llevó a Friedland (2002) a subrayar que ya no solo la ciudad es urbano ni campo lo rural.

La mayoría de los investigadores señalan que no existe una definición aceptada sobre el medio rural y, además, hay una gran diversidad en cuanto a los criterios utilizados para delimitar estos espacios. Las posibles definiciones dependen, en buena medida, de la unidad de análisis, las variables elegidas y la diversidad de los elementos que lo forman (Lázaro, 1995; Ricketts y Johnson-Webb, 1996; Márquez, 2002; Rodríguez, 2005; Scott y Gelan, 2007). Cada vez más en las áreas rurales tienen lugar multiplicidad de actividades y usos que pueden ser considerados urbanos. Por otra parte, se han extendido al medio rural pautas de comportamiento y modos de vida urbanos, lo que provoca que los límites entre lo rural y lo urbano sean más difusos, máxime en el contexto de la Sociedad de la Información y al proceso globalizador en el que vivimos (Esparcia, 2012).

El concepto desarrollo rural expresa y engloba nociones e ideas muy heterogéneas tales como un objetivo de transformación económica, un proceso de cambio, etc. (MAPA, 2003; Ceña, 1995). Siguiendo la propuesta del MAPA (2003) del Gobierno de España, el desarrollo rural puede definirse como un proceso de mejora sostenible y diversificación de su economía basado en la utilización de sus propios recursos, acompañado de una reducción de las desigualdades, bien las que existen entre unas áreas y otras, bien entre el sistema rural y el urbano.

El desarrollo rural, en ocasiones, es visto en la literatura científica como un sinónimo del desarrollo local, aunque tampoco existe para este último, una definición global y ampliamente asumida por los investigadores (Pérez y Carrillo, 2000). Aunque el desarrollo local no lleva asociado de modo exclusivo y directo una referencia territorial de planificación y de gestión de sus programas, ya que puede tener una base rural o urbana, es quizás en las áreas rurales donde el desarrollo local adquiere una mayor presencia e importancia, llegando a confundirse con el desarrollo rural (Rodríguez González, 2004).

La modernización y transformación del sector primario, y en particular de la agricultura, fue la estrategia de desarrollo más seguida en las últimas décadas y en el caso europeo, las políticas de desarrollo rural de la Unión Europea desde sus orígenes así lo avalan. Hasta finales de los años ochenta tuvieron una orientación estrictamente 
sectorial dirigidas a los agricultores y empresas agrícolas con la finalidad de sostener los precios, intervenir en los mercados agrícolas y transformar las estructuras agrarias. En los años noventa y tras comprobar que las medidas adoptadas resultaban insuficientes para fomentar el desarrollo del medio rural debido a su heterogeneidad y diversidad, se diseñaron programas específicos para cada zona con la participación de agentes económicos y sociales con el objetivo de favorecer a la población rural en su conjunto (Saraceno, 2001; Pérez y Caballero, 2003).

A pesar de que existen posiciones en la literatura científica que apuestan por que la agricultura sea el eje central del desarrollo rural, apoyada por otras actividades que contribuyan a impulsar la vida económica y social del campo (Hervieu, 1995), la idea de que la agricultura sea el esqueleto que sostenga el medio rural no es una postura compartida en el seno de la comunidad científica. Lázaro (1995) e Izquierdo (2002) señalan que en la actualidad la agricultura no puede soportar, por sí misma, el tejido socioeconómico local a pesar de que, en el pasado, constituyó la base económica de estas áreas. Los autores subrayan que la revitalización del medio rural debe llevarse a cabo mediante la diversificación de su economía, promoviendo sus recursos endógenos y también atrayendo actividades económicas procedentes del exterior que prefieran localizarse en el rural por razones estratégicas (calidad ambiental, buenas comunicaciones, acceso a banda ancha, incremento de la calidad de vida, etc.).

Siguiendo esta línea, Etxezarreta (1995) hace referencia al papel destacado que puede tener la pluriactividad como factor de dinamización económica y social, fomentando los recursos existentes en un determinado territorio y aprovechando, a su vez, los recursos humanos y financieros para estimular todas aquellas actividades que puedan tener lugar en el medio rural. Y una de las estrategias que adopta este objetivo es el fomento del turismo rural.

El turismo rural es una de estrategias más recurridas para cambiar o reducir la situación de declive que padecen una gran parte de las áreas rurales. Se trata de una actividad complementaria de gran relevancia en la generación de rentas pero, al mismo tiempo, es conveniente tener en cuenta sus limitaciones y riesgos (Cals et al., 1995; Etxezarreta, 1995; Santos, 1999; Izquierdo, 2002). En este sentido, Santos (1999) advierte de las limitaciones del turismo en los procesos de desarrollo rural y apunta que, en buena parte de los programas que se llevaron a cabo, tuvieron el turismo como objetivo prioritario y le fueron dedicados muchos recursos. Se le atribuye al turismo como el gran salvador del medio rural en declive y pese a que son variados los beneficios en las comunidades donde se instala, es necesario tener cautela a la hora de atribuirle una importancia excesiva.

En pleno proceso de inmersión en la Sociedad de la Información, la estrategia que, desde nuestro punto de vista puede tener más peso a la hora de impulsar procesos de desarrollo en el medio rural, son las actividades vinculadas al uso de las nuevas tecnologías y en particular, al consumo de los servicios avanzados de Internet (Armas y Macía, 2015). Las nuevas tecnologías ayudan a reforzar la cohesión económica y social de las regiones, así como a mejorar su competitividad, pero para ello es decisiva la implicación de las administraciones públicas y otros actores económicos (Borges et al., 2007). Existe la convicción generalizada, para impulsar el desarrollo 
económico, de que las tecnologías de la información y de la comunicación son imprescindibles para la atracción de empresas a una determinada localidad o región, del mismo modo que lo fueron en su día los servicios de suministro de agua, electricidad y recogida de basura (Pigg y Crank, 2005).

En gran medida, la situación de declive de una buena parte del medio rural en Europa está motivada por sus condicionantes físicos. La lejanía con respecto a los núcleos urbanos donde se concentraban las principales actividades económicas, y las malas redes de comunicaciones, provocaron la situación de deterioro demográfico y económico que padecen en la actualidad. Pero en la Sociedad de la Información las distancias se diluyen, la comunicación es instantánea y surgen un amplio abanico de servicios y posibilidades que, sabiendo aprovechados, podrán cambiar esa tendencia de declive instaurada en muchas regiones periféricas desde hace décadas.

En la actualidad, y pese a que las políticas de desarrollo rural para el período 20142020 están enmarcadas en la Estrategia Europa 2020, donde se pretende impulsar un crecimiento inteligente, sostenible e inclusivo, las prioridades de estas políticas siguen intensamente orientadas a mejorar la competitividad de las empresas agrarias con una vertiente de conservación y mejora de los ecosistemas dependientes de la agricultura. De las seis prioridades en las que se fundamentan estas políticas, solo una de ellas se centra en fomentar la inclusión social, reducir la pobreza y el desarrollo económico de las áreas rurales. Además, dentro de esta última, tan solo se citan tres iniciativas de carácter muy general, como "promover el desarrollo local en las zonas rurales" o "facilitar la diversificación, la creación y el desarrollo de pequeñas empresas y la creación de empleo" (UE, 2013), sin concretar cuáles podrían ser las medidas para llevarlas a cabo.

\section{La renovación conceptural de la brecha digital}

La revolución de las tecnologías de la información y de la comunicación, que está teniendo como resultado la configuración de un nuevo modelo de sociedad, no es un proceso homogéneo y su trascendencia es muy desigual en función de su grado de difusión en un territorio y su asimilación por parte de su sociedad. Estas diferencias se aprecian no solo entre regiones del mismo país, sino también entre barrios de una misma ciudad (Van der Meer y Van Winden, 2003). Estas disparidades llevaron a algunos autores a hablar del término en plural "Sociedades de la Información" para así poder simbolizar las diferencias entre países y nacionalidades (Steinfeld y Salvaggio, 1989).

Estas desigualdades dieron origen al concepto conocido por la "brecha digital". Con este concepto se pretendía visibilizar la marginación de ciertos territorios y ciudadanos de la Sociedad de la Información. Esta brecha digital inicial, separaba a aquellos territorios que no tenían las infraestructuras para poder acceder a Internet y a aquella población que no la usaba, bien fuese porque no tenía el equipamiento para ello, la formación o ambas cosas. Pero pronto diversos investigadores ampliaron dicho concepto distinguiendo entre las personas que, pese a tener acceso a la Red no lo 
hacían. Lois et al. (2010) distinguieron dentro del grupo de individuos que tenían acceso a Internet, los que realmente la utilizaban (usuarios reales) y aquellos que pese a tener la posibilidad de hacerlo no se conectaban (usuarios potenciales).

Las investigaciones sobre el fenómeno de la brecha digital comenzaron con la observación de las personas que tenían a su disposición un ordenador y conexión a Internet, y aquellas que no tenían esta posibilidad. Hoy día, una buena parte de los investigadores que estudian este fenómeno se centran en el acceso físico, pero en los últimos años algunos autores sugirieron la necesidad de dar un paso más sobre los aspectos sociales y culturales que motivan esas desigualdades (Van Dijk, 2006). Otros incluso apuntan que ciertos grupos sociales tendrán muy pocas posibilidades de llegar a usar estas tecnologías (Bromley, 2004; Roe y Broos, 2005).

Es recurrente la importancia de variables tales como la edad, estatus socioeconómico, educación, composición familiar, etc. a la hora de utilizar las nuevas tecnologías (DiMaggio et al., 2004; Selwyn, 2006; Van Dijk, 2006; Dutton et al., 2011; Van Deursen y Van Dijk, 2014). Tener acceso a las tecnologías no significa que se usen y, además, ni su utilización es homogénea ni siempre genera productividad. Algunos usuarios son capaces de utilizar estas herramientas de forma continua, comprensiva y provechosa, pero en otras ocasiones, ese uso es muy limitado y espasmódico (Wilhelm, 2000).

En el proceso de inmersión en la Sociedad de la Información, la motivación del individuo es una condición básica y que influye de forma notable en la decisión de comprar un ordenador, conectarse a Internet, adquirir conocimientos para "navegar" y comunicarse, además de utilizar aplicaciones variadas (Van Dijk, 2005). Las razones para esa falta de motivación son múltiples entre las que podrían estar no necesitarlas, falta de tiempo, dinero, formación, etc. Muchas de las personas que no usan las nuevas tecnologías, tienen problemas motivacionales bien sea de naturaleza social, cultural, mental o psicológica (Van Dijk, 2006). Una explicación social importante podría ser que Internet no resulta a atractivo a personas con bajos ingresos y nivel educativo (Katz y Rice, 2002).

La brecha digital tradicional o en el acceso, debe ser reevaluada pues está dando paso a una nueva brecha digital en los diferentes usos de la Red (Bucy, 2000; Gunkel, 2003; Peter y Valkenburg, 2006; Selwyn, 2006; Van Deursen y Van Dijk, 2010; Armas y Macía, 2015). Si la brecha digital tradicional o en el acceso separaba a los ciudadanos que usaban las nuevas tecnologías de los que no lo hacían, en este caso, la nueva brecha digital de segunda generación separa a los usuarios que utilizan la Red casi en exclusiva para consultar información y comunicarse, de los que la utilizan de una forma mucho más amplia, consumiendo servicios avanzados de Internet como el comercio electrónico, la formación en línea, la banca electrónica, la administración electrónica, el trabajo a distancia, etc. (Piñeira et al., 2014). Entre los servicios que están disponibles en Internet, existen actividades y aplicaciones que son mucho más provechosas que otras y que pueden suponer una mejora y avances desde muchos puntos de vista, tales como en la carrera profesional, la formación, mejoras laborales, etc. (Hargittai y Hinnant, 2008). 
Los cambios que están teniendo lugar en la sociedad actual demandan nuevas habilidades y conocimientos en el manejo de Internet, y de existir muchas diferencias entre la población en el conocimiento de dichas habilidades, podrían generarse consecuencias negativas e incrementar las desigualdades sociales (Van Dijk, 2005; Witte y Mannon, 2007). La gran expansión que tuvo las comunicaciones móviles (Smartphones, Tables, etc.), así como la ubicuidad de la Red, hace necesario estudiar de forma más profunda nuevas brechas digitales potenciales (Peter y Valkenburg, 2006; Hassan, 2008).

Desde el punto de vista sociológico, existen pocos estudios que aborden el tema de las habilidades en el manejo de Internet, y los que existen, suelen utilizar encuestas donde se les pregunta a los entrevistados acerca de cuáles son sus habilidades en el manejo de esta Red. A este respecto, Van Deursen y Van Dijk (2010) señalan que la brecha digital es más grande cuanto mayor es la brecha en el acceso. Es evidente que este fenómeno aún está presente en las regiones más periféricas y en algunos sectores sociales, como pueden ser las personas de mayor edad o la población con muy baja cualificación, que no perciben la utilidad de las nuevas tecnologías, pero que son valores muy modestos en relación a la población "conectada" (Armas y Macía, 2015).

La brecha digital tradicional ha dado paso a una nueva brecha de segunda generación, y esta sí que tiene una importante presencia y es más profunda en aquellos territorios donde la brecha en el acceso ya tenía más incidencia, como es el caso de las áreas rurales periféricas. Mientras que la brecha digital tradicional es más fácil de superar, la nueva brecha digital de segunda generación, que separa a los usuarios de Internet que tienen diferentes niveles de habilidades, se prevé que sea mucho más complicada de salvar (Van Deursen y Van Dijk, 2010; Armas y Macía, 2015).

Existen varios estudios que han afrontado el reto de medir las habilidades que posee una determinada población en el manejo de Internet utilizando diferentes indicadores. Uno de ellos es el de Steyaert (2002) que distinguió entre habilidades instrumentales, habilidades estructurales, y habilidades estratégicas. Las primeras son las que se relacionan con la propia manipulación de la tecnología, las segundas con la estructura en la cual está contenida la información, y las habilidades estratégicas son aquellas que le permiten al ciudadano buscar información relevante para la toma de decisiones y las que podrían contribuir a superar la brecha digital de segunda generación.

En esta línea, Van Deursen y Van Dijk (2010) también distinguieron cuatro tipos de habilidades en el uso de la Red: habilidades operacionales, habilidades formales, habilidades de información, y habilidades estratégicas. Según estos autores, los dos primeros grupos que se corresponden con los usos básicos, no son suficientes para conseguir un uso eficiente de Internet. En los dos últimos grupos, los usuarios tienen la capacidad de encontrar, seleccionar, procesar y avaluar la información procedente de diferentes fuentes, además de usar esa información de manera que sirva para conseguir una serie de objetivos y que ello implique una mejora en la posición social.

Otros investigadores, como Piñeira et al. (2014) hacen al respecto una distinción más simple, dividiendo las habilidades de los usuarios de Internet en dos grupos. En el primero los ciudadanos hacen un uso muy simple de la Red, como comunicarse con 
otros usuarios, bien sea a través del correo electrónico o de las redes sociales, buscar y consultar información, descarga de recursos multimedia, o simplemente "navegar" sin ningún fin en particular. En el segundo los ciudadanos "consumen" de los servicios avanzados de la Red y los utilizan para formarse (e-learning), para trabajar a distancia (teleworking), para contratar servicios, comprar y vender bienes (e-commerce), para tramitar y realizar gestiones con la administración pública o con las entidades bancarias (e-administration y e-banking), toda una serie de tareas que mejoran la calidad de vida de los ciudadanos. Son estos los servicios de Internet los que posibilitan una plena inmersión en la Sociedad de la Información y, a su vez, los que pueden impulsar procesos de desarrollo en el medio rural. La innovación es un elemento clave para promover el desarrollo sostenible y la competitividad de un territorio y depende del acceso a información codificada (Borges et al., 2007). Es por tanto necesario, impulsar el uso de las habilidades estratégicas (Steyaert, 2002; Van Deursen y Van Dijk, 2010) y de los servicios avanzados de Internet (Piñeira et al., 2014).

\section{Los Servicios Avanzados de Internet: oportunidades de desarrollo para el medio rural}

La difusión de los servicios avanzados resulta imprescindible para lograr una plena inmersión en la Sociedad de la Información dado que la utilización básica de la Red, no supondrá avances considerables más allá de la simple comunicación y consulta de información. Las primeras plataformas de Internet, tales como la World Wide Web (WWW), han estado centradas en las comunicaciones entre individuos y grupos de personas. El consumo de servicios avanzados de Internet abrirá al camino a la Internet de las Cosas, donde será posible la comunicación y gestión entre personas y dispositivos y también la comunicación entre distintos dispositivos (Chen, Wan y Li, 2012). El desarrollo de la Internet de las Cosas implicará cambios considerables desde el punto de vista social, tecnológico y científico ya que se incorporarán elementos nuevos a la Sociedad de la Información (UIT, 2015).

El volumen de datos generados en formato digital se duplica cada dos años (EMC, 2014) debido a la gran cantidad de plataformas y servicios que existen y que se crean día a día en Internet. Este hecho manifiesta la tendencia y necesidad a utilizar la Red de una forma más amplia, más allá de consultar información y comunicarse. Dado que la adopción de las nuevas tecnologías está siendo más rápida en las áreas urbanas, donde las "brechas digitales" son menos profundas, según la UIT (2015), el impacto de los servicios avanzados y la Internet de las Cosas será mayor en estos, sobre toto en el área de la salud, los transportes, y la consolidación de las Smart Cities.

La difusión de los Smartphones, y en especial la conexión a Internet a través de estos dispositivos fue muy importante en los últimos años fruto de la instalación de redes de telecomunicaciones móviles. Este hecho motivó que el número de usuarios de Internet se triplicase en los últimos diez años lo que significa que, en la actualidad, casi el $44 \%$ de la población del mundo está conectada a la Red de redes. Los 
Smartphones y las Tablets están desplazando a los teléfonos móviles básicos y a los ordenadores personales tradicionales y los usuarios demandan, cada vez más, una conectividad más móvil y rápida, así como los crecientes servicios que ofrece la Red.

Pero los avances logrados en los últimos años en cuanto a banda ancha móvil y usuarios de Internet, no son suficientes para lograr una inserción plena en la Sociedad de la Información. La mayor parte de los individuos que se han unido a las nuevas tecnologías, son internautas que hacen un uso de la Red muy básico y que se restringe a la consulta de información, la comunicación a través de correo electrónico y las redes sociales, y un número muy reducido de servicios. En la Unión Europea (UE-28), donde ocho de cada diez ciudadanos son usuarios de Internet, casi el 90\% de ellos la utiliza Internet para enviar correos, la mitad la usa para hacer compras y solo un $7 \%$ hace cursos en línea. Es esta nueva brecha digital la que debe ser superada para lograr una plena inserción en la Sociedad de la Información, especialmente en las áreas rurales más periféricas.

Para afrontar la nueva brecha digital de segunda generación es necesario impulsar el uso de los servicios avanzados de Internet y preparar así el camino para la futura Internet de las Cosas. Los servicios avanzados forman parte de la rutina diaria de muchos ciudadanos que utilizan la Red para comprar bienes o contratar servicios, gestionar operaciones bancarias, hacer trámites con la administración pública, o formarse. Todas estas tareas dinamizan procesos, ahorran tiempo y dinero, y pueden contribuir a iniciar procesos de desarrollo. Dos de los servicios avanzados de Internet que pueden llegar a tener un mayor impacto en las áreas rurales son el comercio electrónico y el trabajo a distancia.

Tanto el comercio electrónico como el trabajo a distancia pueden contribuir a promover procesos de desarrollo en el medio rural, mitigando o invirtiendo las tendencias sociales y económicas regresivas que afectan a buena parte de estos espacios (Blanco y Cánoves, 1998; 1999; Grimes, 2000; Richardson y Gillespie, 2003; Simpson et al., 2003). El trabajo a distancia puede ser una alternativa para crear un espacio en el mercado laboral e integrar a la población más joven y cualificada que otrora se veía obligada a emigrar a la ciudad, y el comercio electrónico puede llegar a convertirse en una posibilidad de negocio y generación de empleo en el medio rural.

El comercio electrónico se presenta como una gran oportunidad para los espacios rurales, tanto en los países desarrollados como en los que están en vías de desarrollo o subdesarrollados. En la Sociedad de la Información, al desaparecer las distancias, las empresas emplazadas en regiones periféricas tienen la posibilidad de ofrecer sus productos y servicios a cualquier parte del planeta, pasando de actuar en un mercado local, limitado, a competir en el mercado global. Cada día son más los ciudadanos que compran y venden a través de la Red y su evolución se puede constatar en la Unión Europea (UE-28), donde en poco más de un lustro, se duplicaron los internautas que hicieron uso de este servicio. Drucker (2001) afirma que el comercio electrónico será, en la Sociedad de la Información, lo que en su día fue el ferrocarril en la Revolución Industrial. Se trata de una revolución nueva y sin precedentes que provocará cambios rápidos en la economía y por ello, las áreas rurales deben saber aprovechar esta oportunidad. 
El trabajo a distancia surge como una nueva organización de la actividad laboral en el contexto de la Sociedad de la Información. Permite llevar a cabo el trabajo desde el domicilio familiar, en los desplazamientos laborables tales como aeropuertos u hoteles (teletrabajo móvil), o centros satélite diseñados para el trabajo a distancia y localizados de forma estratégica para reducir los desplazamientos de los trabajadores (Martínez et al., 2006). Este servicio avanzado de Internet puede significar, para las áreas rurales, nuevas posibilidades de cara a fijar población y reactivar el desarrollo económico y social. El trabajo a distancia va a diluir la delimitación entre el trabajo y el hogar, pasando este a ser un lugar de convivencia de muchos aspectos de la vida humana donde el relax, el ocio y descanso, dejan de ser exclusivos e incorporan nuevas funciones como lugar de trabajo, formación, etc. (Cairncross, 2001).

Pese a que el término "Internet de las Cosas" está siendo cada día más utilizado, no existe aún una definición aceptada sobre lo que este término abarca. La Unión Internacional de Telecomunicaciones la define "como una estructura global para la Sociedad de la Información, que propicia la prestación de servicios avanzados mediante la interconexión de objetos (físicos y virtuales) gracias a la interoperabilidad de las tecnologías de la información y de la comunicación" (ITU, 2012). Al mismo tiempo, surgieron multitud de definiciones alternativas. Algunas de ellas inciden en las cosas o dispositivos que pueden llegar a ser conectados, otras se centran en aspectos como los protocolos de Internet o las tecnologías de redes. Y un tercer grupo de definiciones abordan desafíos semánticos de la Internet de las Cosas relacionado con el almacenamiento, búsqueda y organización de grandes cantidades de información (Atzori et al., 2010).

El surgimiento de la Internet de las Cosas representa para Roselli et al. (2015) una evolución de las propias tecnologías de la información, siendo estas un factor clave e indispensable sin las cuales la Internet de las Cosas no podría existir. Las nuevas tecnologías son imprescindibles para la transmisión y divulgación de datos, así como para facilitar de forma eficiente los distintos servicios a la sociedad. El hecho de que la Internet de las Cosas se centre en conectar dispositivos que abarcan una gran cantidad de posibilidades (transporte, comercio, agricultura, educación, salud, hogares, industria), no significa que ello sea el límite de esta evolución tecnológica. Investigadores y expertos prevén una evolución más de esta tecnología, pasando de la Internet de las Cosas a la "Internet de lo Todo" (Internet of Everything) donde las comunicaciones entre personas, dispositivos, datos y procesos, estarán totalmente unificados (Bradley et al., 2013).

La Internet de las Cosas probablemente revolucionará la forma en que individuos, corporaciones, instituciones y gobiernos se relacionen con el mundo. Del mismo modo, ofrecerán también nuevas posibilidades de desarrollo al proveer fuentes de datos que podrían contribuir a entender, analizar y abordar muchos aspectos relacionados con los procesos de desarrollo. Debido a ello, se está generando un debate en relación a las posibilidades que ofrece para impulsar procesos de desarrollo (UIT, 2015).

La rápida evolución que está experimentando día a día el sector de las tecnologías de la información y de la comunicación, así como a la reducción del coste de los 
dispositivos, del acceso a Internet, y la gran difusión de la banda ancha (bien sea fija o móvil), suponen una gran oportunidad de desarrollo para el medio rural. Son necesarias actuaciones para impulsar el uso de servicios avanzados, tales como el comercio electrónico y el trabajo a distancia, ya que estos pueden reducir, e incluso invertir, su dinámica regresiva. Un simple uso de la Red no genera conocimiento ni impulsa procesos de desarrollo, por lo que la nueva brecha digital de segunda generación, debe ser superada para poder aprovechar al máximo sus potencialidades y preparar así el camino hacia de la Internet de las Cosas.

\section{Reflexiones finales}

La revolución de las tecnologías de la información y de la comunicación, iniciada ya hace varias décadas, provocó importantes cambios en la sociedad y en la economía, pero estos cambios serán cada vez más intensos conforme se generalice el uso de los servicios avanzados de Internet y se instaure la futura Internet de las Cosas. Todos estos cambios también están afectando a las áreas rurales, aunque su impacto es tan desigual como su diversidad. Para estudiar estos cambios y la situación real de cada territorio, son necesarias delimitaciones urbano-rurales más precisas que superen la utilización, casi en exclusiva, de criterios demográficos. Establecer varios rangos definitorios específicos en el medio rural, ayudará a diseñar mejor las estrategias y programas de desarrollo a seguir en cada caso.

Los programas de desarrollo rural del siglo XXI deben ir más allá de las actuaciones en el sector primario. Pese a que en el pasado fue el motor económico y el sustento de las sociedades rurales, en la actualidad y en concreto en Europa, su peso en la economía y en el empleo decreció de forma considerable dando paso a otras actividades económicas, fundamentalmente vinculadas al sector de los servicios. Las políticas de desarrollo del medio rural deben gravitar en la pluriactividad, buscando iniciativas que impulsen un crecimiento inteligente, sostenible e inclusivo, tal y como señala la Estrategia Europa 2020 y que parece que desoyen las políticas de desarrollo rural de la Unión Europea para el período 2014-2020.

Tal y como se constató en la revisión de la literatura científica, las tecnologías de la información y de la comunicación son imprescindibles para la atracción de empresas, como lo fue en su día los servicios de suministro de agua y recogida de basura. El medio rural tiene nuevas oportunidades de desarrollo en la Sociedad de la Información, pero para ello es necesario hacer un uso "inteligente" de la Red e ir más allá de los servicios básicos de comunicación y consulta de información. Dada la gran cantidad de información y servicios que existen en la Red, creciendo día a día a pasos agigantados, el grado de conocimiento y habilidades de Internet es vital para impulsar procesos de desarrollo.

Los servicios avanzados de Internet, tales como el comercio electrónico, el trabajo a distancia, la formación en línea, la banca y la administración electrónica, suponen para los ciudadanos, no solo un incremento en su calidad de vida, sino también oportunidades de negocio y empleo. Muchas de estas tareas, que ahorran tiempo y 
dinero en desplazamientos, pueden contribuir en el medio rural a impulsar procesos de desarrollo. En Europa la brecha digital tradicional puede darse por superada y los esfuerzos ahora deben estar centrados en vencer la nueva brecha digital de segunda generación, fomentando el uso de los servicios avanzados, preparándose así para la futura Internet de las Cosas.

\section{Bibliografía}

Armas Quintá, F.X.; Macía Arce, X.C. (2015). Da cidade dixital á cidade do coñecemento. Un proceso inacabado. En: Lois, R.C.; Pino, D. A Galicia Urbana. Vigo: Edicións Xerais de Galicia, pp. 449-462.

Armesto López, X.A. (2005). Notas teóricas en torno al concepto de postproductivismo agrario. Investigaciones Geográficas, 36, pp. 137-156.

Atzori, L.; Lera, A.; Morabito, G. (2010). The Internet of Things: A survey. Computer Networks, 54, pp. 2787-2805.

Bell, D. (1974). The coming of Post-Industrial Society. Londres: Heinemann.

Bericat Alastuey, E. (1993). La teoría del vacío rural. En: Junta de Andalucía, Consejería de Agricultura y Pesca. El desarrollo rural a las puertas del siglo XXI. Sevilla: Junta de Andalucía, pp. 45-54.

Borges Tiago, M.; Couto, J.; Dos Santos Natário, M.; Martíns Braga, A. (2007). The adoption of communication and information technologies and the local development. Journal of Business Economics and Management, 8 (2), pp. 111-117.

Bradley, J.; Reberger, C.; Dixit, A.; Gupta, V. (2013). Internet of Everything: A \$4.6 Trillion Public-Sector Opportunity, CISCO Internet Business Solutions Group. White Paper.

Bromley, C. (2004). Can Britain close the digital divide? In: Park, A.; Curtice, J.; Thomson, K.; Bromley, C.; Philips, M. (eds.). British Social Attitudes: The 21st Report. London: Sage, pp. 73-99.

Bucy, E.P. (2000). Social access to the Internet. The International Journal of Press/Politics, 5 (1), pp. 50-61.

Cairncross, F. (2001). The death of distance 2.0. How the communications revolution will change our lives. London: Texere, $317 \mathrm{p}$.

Cals, J.; Capellá, J.; Vaqué, E. (1995). El turismo en el desarrollo rural de España. Madrid: Ministerio de Agricultura, Pesca y Alimentación, $122 \mathrm{p}$.

Camarero, L. (1993). Del éxodo rural y del éxodo urbano. Madrid: Ministerio de Agricultura, Pesca y Alimentación.

Castells, M. (2000). La era de la información: economía, sociedad y cultura. La sociedad red. Madrid: Alianza Editorial.

Ceña Delgado, F. (1995). Planteamientos económicos del desarrollo rural: una perspectiva histórica. En Ramos, E., Cruz, J. (coords.). Hacia un nuevo sistema rural. Madrid: Ministerio de Agricultura, Pesca y Alimentación, pp. 91-129.

Champion, T.; Shepherd, J. (2006). Demographic change in rural England. In: Lowe, P.; Speakman, L. (Eds.). The Ageing Countryside. London: Age Concern England. 
Chen, M.; Wan, J.; Li, F. (2012). Machine-to-Machine Communications. KSII Transactions on Internet and Information Systems (TIIS). 2, pp. 480-497.

Cloke, P. (1977). An index of rurality for England and Wales. Regional Studies, 11, pp. 31-46.

Cloke, P.; Goodwin, M. (1993). Rural change: structured coherence or unstructured incoherence? Terra, 105 (3), pp. 166-174.

Cloke, P. (2006). Conceptualizing rurality. In: Cloke, P., Marsden, T., Mooney, P. (Eds.) The Handbook of Rural Studies. London: SAGE Publications, pp. 18-28.

Clout, H. (1993). European experience of rural development. London: Rural Development Commission.

Dimaggio, P.; Hargittai, E.; Celeste, C.; Shafer, S. (2004). From unequal access to differentiated use: a literature review and agenda for research on digital inequality. In: Neckerman, K. (ed.). Social Inequality. New York: Russell Sage Foundation, pp. 355-400.

Druker, P. (2001). Detrás de la revolución de la información. La Factoría. Disponible en Internet: http://www.lafactoriaweb.com/articulos/drucke13.htm.

Dutton, W.; Helsper, E.; Gerber, M. (2011). The Internet in Britain in 2011. Oxford: Oxford Internet Institute.

EMC (2014). Digital Universe Study [consulta: 2 de septiembre de 2016]. Disponible en Internet: http://www.emc.com/leadership/digital-universe/index.htm?pid=landingdigitaluniverse-131212.

Entrena-Durán, F. (2012). La ruralidad en España: de la mitificación conservadora al neorrularismo. Cuadernos de desarrollo rural 9(69), 39-65.

España. Ministerio De Agricultura, Pesca y Alimentación. (2003). Libro Blanco de la agricultura y el desarrollo rural. Madrid: Ministerio de Agricultura, Pesca y Alimentación, 4 vols.

Esparcia, J. (2012). Evolución reciente, situación actual y perspectivas futuras en el desarrollo rural en España y en la UE. Revue Marocaine d'Administration Locale et de Développement 79, 53-84.

Etxezarreta, M. (1995). Una visión crítica de las políticas agrarias españolas en el contexto internacional. En: Ramos, E.; Cruz, J. (coords.). Hacia un nuevo sistema rural. Madrid: Ministerio de Agricultura, Pesca y Alimentación, p. 169-202.

Florencio Calderón, A. (2000). El desarrollo local y el desarrollo rural: el papel de los pequeños municipios. En: Pérez Ramírez, B.; Carrillo Benito, E. (coords.). Desarrollo local: manual de uso. Madrid: ESIC, p. 527-554.

Friedland, W.H. (2002). "Agriculture and rurality. Beginnig the final separation?" Rural Sociology 67(3), pp. 350-371.

García Ramón, M.D., Tulla I Pujol, A.F., Valdovinos Perdices, N. (1995). Geografía Rural. Madrid: Síntesis.

García Sanz, B. (1996). La sociedad rural ante el siglo XXI. Madrid: Ministerio de Agricultura, Pesca y Alimentación.

Gunkel, D. J. (2003). Second thoughts: toward a critique of the digital divide. New Media \& Society. 5, pp. 499-522.

Halfacree, K. (1993). Locality and social representation: space, discourse and alternative definitions of the rural. Journal of Rural Studies, 9 (1), pp. 23-27. 
Hargittai, E.; Hinnant, A. (2008). Digital inequality: differences in young adults' use of the Internet. Communication Research, 35 (5), pp. 602-621.

Hassan, R. (2008). The Information Society. Cambridge (MA): Polity Press.

Hewitt, M. (1989). Defining rural areas. Impact on health care policy and research. Washington D.C.: United States Government Printing Office.

Hoggart, K. (1990). Let's do away with rural. Journal of Rural Studies, 6 (3), pp. 245-257.

International Telecommunication Union. (2015). Measuring the Information Society Report. Geneva, Switzerland, 234 pp.

Izquierdo Vallina, J. (2002). Manual para agentes de desarrollo rural. Madrid: Instituto de desarrollo rural, $453 \mathrm{p}$.

Katz, J. E.; Rice, R. E. (2002). Social consequences of Internet Use, Access, Involvement and Interaction. Cambridge, MA: MIT Press.

Lázaro Araujo, L. (1995). El desarrollo rural en el contexto de la Unión Europea. En: Ramos, E., Cruz, J. (coords.). Hacia un nuevo sistema rural. Madrid: Ministerio de Agricultura, Pesca y Alimentación, pp. 237-255.

Lois González, R. C.; Macía Arce, J. C.; Armas Quintá, F. J. (2010). ICT inequalities in the Spanish urban system. Journal of Urban and Regional Analysis. 2 (2), pp. 19-32.

Lois González, R.C. (2014). As transformacións recentes nun rural galego que mudou. En: Saavedra Fernández, P., Rodríguez González, R. (Dir.). A terra en Galicia: pasado presente e futuro. Granada: Universidade Internacional Menéndez Pelayo; Lalín: Concello de Lalín, pp. 112-137.

Márquez Fernández, D. (Coord.) (2002). Nuevos horizontes en el desarrollo rural. Madrid: Akal.

Martínez Sánchez, A.; Pérez Pérez, M; De Luis Carnicer, P.; Vela Jiménez, M. (2006). Trabajo y flexibilidad: efecto moderador sobre los resultados de la empresa. Cuadernos de Economía y Dirección de la Empresa. 29, pp. 229-262.

Moyano, E. (2000). Procesos de cambio en la sociedad rural española: Pluralidad de intereses en una nueva estructura de oportunidades. Papers: Revista de Sociología. 61, pp. 191-220.

Muilo, T. (2010). Needs for rural research in the northern Finland context. Journal of Rural Studies, 26(1), 73-80.

Paniagua Mazorra, A.; Hoggart, K. (2002). Lo rural, ¿Hechos, discursos o representaciones? Una perspectiva geográfica de un debate clásico. Información Comercial Española. Revista de Economía, 803, pp. 61-71.

Pérez Ramírez, B.; Carrillo Benito, E. (coords.) (2000). Desarrollo local: manual de uso. Madrid: ESIC. 676 p.

Pérez, A.; Caballero, J.M. (2003). La nueva ruralidad en Europa y su interés para América Latina. Roma: FAO. Disponible en Internet: http://www.fao.org/docrep/004/y4524s/y4524s00.HTM.

Peter, J.; Valkenburg, P.M. (2006). Adolescents' internet use: Testing the "disappearing digital divide" versus the "emerging digital differentiation" approach. Poetics 34, pp. 293-305.

Pick, J. B.; Azari, R. (2008). Global digital divide: Influence of socioeconomic, governmental and accessibility factors on information technology. Information Technology for Development, 14(2), pp. 91-115. 
Pigg, K.; Crank, L. (2005). Do Information Communication Technologies promote rural economic development? Community Development, 36 (1), pp. 65-76.

Piñeira Mantiñán, M.J.; Armas Quintá, F.J.; Macía Arce, J.C. (2014) El consumo de servicios avanzados de Internet en el proceso de configuración de las "Smart Cities". Estudios de caso en Galicia y Madrid. Cuadernos Geográficos, 53(2), pp. 103-134.

Prados, M.J. (2009). Naturbanization: New identities and processes for rural-natural areas. In: Prados, M.J. (ed.). Naturbanization: New identities and processes for rural-natural areas. London: Taylor \& Francis Group, pp. 3-7.

Ricketts, T.C.; Johnson-Webb, K.D. (1996). What is rural and How to Measure Rurality. A Focus on Health Care Derively and Health Policy. Chapel Hill, North Carolina: University of North Carolina.

Roca, J. (2003). “La delimitación de la ciudad: ¿una cuestión imposible?” Ciudad y Territorio. Estudios Territoriales, 35 (135), pp. 17-36.

Rodríguez González, R. (2004). Xeografia, entre cultura e profesión territorial. Vigo: Ir Indo. $197 \mathrm{p}$.

Rodríguez Rodríguez, M. (2005). Revisión de las políticas de desarrollo rural y su incidencia en los municipios rurales de las provincias de Almería. Los servicios de proximidad como base para su reformulación. Málaga: Analistas Económicos de Andalucía-Fundación Unicaja.

Roe, K.; Broos, A. (2005). Marginality in the information age: the socio-demographics of computer disquietude. European Journal of Communication, 30(1), pp. 275-277.

Roselli, L.; Mariotti, C.; Mezzanotte, P.; Alimenti, F.; Orecchini, G.; Virili, M.; Carvalho, N.B. (2015). Review of the Present Technologies Concurrently Contributing to the Implementation of the Internet of Things (IoT) Paradigm: RFID, Green Electronics, WPT and Energy Harvesting. 2015 IEEE Topical Conference Wireless Sensors and Sensor Networks (WiSNet), IEEE Topical Conference.

Rubio Terrado, P. (2010). Modelización de los cambios y evolución reciente del sistema rural español. Boletín de la Asociación de Geógrafos Españoles, 54, pp. 203-235.

Sancho Comíns, J.; Reinoso Moreno, D. (2012). La delimitación del ámbito rural: una cuestión clave en los programas de desarrollo rural. Estudios Geográficos, 73(273), pp. 599-624.

Sancho Hazak, R. (1997). Estructura demográfica y tipificación de los asentamientos y áreas rurales españolas. En: GÓMEZ BENITO, C., GONZÁLEZ RODRÍGUEZ, J. Agricultura y sociedad en la España Contemporánea. Madrid: Ed. CIS, pp. 173-224.

Santos Solla, X.M. (1999). Reflexións entorno ó papel do turismo no desenvolvento local. En: Actas Seminario Internacional sobre perspectivas de desarrollo en Iberoamérica, (Santiago de Compostela, 3-7 de mayo de 1999). Santiago de Compostela: Universidade de Santiago de Compostela, pp. 149-165.

Saraceno, E. (2001). La experiencia Europea de Desarrollo Rural y su utilidad para el contexto Latinoamericano. En: Seminario Internacional Agricultura Familiar y Desarrollo Sustentable, Brasilia, EMBRAPA, 21 a 23 de noviembre 2001.

Scott, A.; Gilbert, A.; Gelan, A. (2007). The Urban-Rural Divide: Myth or Reality? SERG Policy Brief, $n^{\circ} 2$. Aberdeen, Scotland (UK): The Macaulay Institute.

Selwyn, N. (2006). Digital division or digital decision? A study of non-users and low-users of computers. Poetics, 34, pp. 273-292. 
Steinfeld, C; Salvaggio, J. L. (1989). Toward a definition of the Information Society. In: Salvaggio, J. L. (ed.). The Information Society: Economic, Social and Structural Issues. Hillsdale (NJ): Lawrence Erlbaum, pp. 1-14.

Steyaert, J. (2002). Inequality and the digital divide: Myths and realities In: HICK, S.; Mcnutt, J. (eds.). Advocacy, Activism and the Internet. Chicago, IL: Lyceum Press, pp. 199-211.

Strover, S. 2003. Remapping the digital divide. The Information Society, 19, pp. 275-277.

Unión Europea (2013). Reglamento (UE) n 1305/2013 del Parlamento Europeo y del Consejo de 17 de diciembre de 2013 relativo a la ayuda al desarrollo rural a través del Fondo Europeo Agrícola de Desarrollo Rural (Feader) y por el que se deroga el Reglamento (CE) n o 1698/2005 del Consejo. Diario Oficial de la Unión Europea, L 347/487, 20 de diciembre de 2013, pp. 487-548.

Unión Internacional De Telecomunicaciones (2012). Recomendación UIT-T T.2060. Serie Y: Infraestructura mundial de la información, aspectos del protocolo Internet y redes de la próxima Generación, Ginebra, Suiza.

Van Der Meer, A.; Van Winden, W. (2003). E-governance in Cities: A Comparison of Urban Information and Communication Technology Policies. Regional Studies, 37(4), pp.407-419.

Van Deursen, A.; Van Dijk, J. (2010). Internet Skills and the digital divide. New Media \& Society 13(6), pp. 893-911.

Van Deursen, A., Van Dijk, J. (2014). The digital divide shifts to differences in usage. New Media \& Society, 16(3), pp. 507-526.

Van Dijk, J. (2005). The Deepening Divide. Inequality in the Information Society. London: Sage Publications, 248 pp.

Van Dijk, J. (2006). Digital Divide: Research, achievements and shortcomings. Poetics, 34, pp. 221-235.

Ward, N.; Brown, D. (2009). Placing the rural in regional development. Regional Studies, 43(10), pp. 1237-1244.

Wilhelm, A. (2000). Democracy in the Digital Age: Challenges to Political Life in Cyberspace. New York: Routledge.

Witte, J.; Mannon, S. (2007). The Internet and Social Inequalities. New York: Routledge.

Woods, M. (2011). Rural. Key Ideas in Geography. Abingdon: Routledge, 336 p. 